\title{
Évolution de la teneur en composés
} aromatiques des aérosols urbains au cours d'une journée. Étude par spectrométrie de masse à deux lasers

\author{
O.P. Haefliger, T.D. Bucheli et R. Zenobi
}

Laboratoire de Chimie Organique, École Polytechnique Fédérale de Zurich (ETH), Universitätstrasse 16, 8092 Zurich, Switzerland

\begin{abstract}
Laser desorption/laser photoionization mass spectrometry (two-step laser mass spectrometry, L2MS) is a powerful tool for the selective analysis of particle-bound organic compounds. After infrared laser-induced thermal desorption, the analytes are photoionized by an ultraviolet laser pulse. The ions are then mass separated and detected in a time-of-flight mass spectrometer. The main feature of this analytical method is an excellent sensitivity, allowing the direct analysis of very small quantities of particles collected on a filter. This allowed us to follow the temporal development of selected polycyclic aromatic hydrocarbons on urban aerosol samples with only 15 minutes time resolution. Data obtained over the course of one day in February 1998 is presented. The temporal behavior was found to be quite different for different mass spectral peaks, which may be due to different emission sources.
\end{abstract}

1 est admis aujourd'hui que les aérosols atmosphériques jouent un rôle essentiel dans la chimie atmosphérique [1] et qu'ils représentent un important facteur de risques pour la santé [2]. Pour cette raison, des recherches visant à caractériser les particules qui les constituent sont actuellement menées dans diverses directions. Les paramètres les plus souvent étudiés sont leur concentration, la distribution de leurs tailles, ainsi que leur composition élementaire [3]. Une description plus complète des particules, notamment de la réactivité chimique à leur surface, de leurs propriétés toxicologiques ou de leurs coefficients d'adhérence, exige cependant que l'on dispose également d'une analyse chimique détaillée des composés organiques adsorbés à leur surface.

L'analyse chimique quantitative est généralement effectuée par des méthodes chromatographiques [voir par exemple 4 ou 5]. Ces techniques nécessitent toutefois une préparation fastidieuse des échantillons ainsi que des quantités d'aérosol relativement grandes. Comme la récolte des échantillons s'effectue donc sur des périodes relativement longues, typiquement un jour complet, les résultats expérimentaux représentent des moyennes des différentes valeurs observées durant cet intervalle de temps. Ils ne reflètent par conséquent pas du tout la variabilité des différentes substances au cours de la journée.

Dans le but de permettre une meilleure compréhension du comportement dynamique des aérosols atmosphériques, nous avons cherché une méthode qui permette d'effectuer des mesures couvrant des périodes plus courtes. La spectrométrie de masse à deux lasers (L2MS) [6] s'est ainsi imposée pour étudier l'évolution au cours d'une journée de la teneur en composés aromatiques polycycliques (PACs) des particules d'aérosols urbains.

La figure 1 explique le principe de la L2MS. Cette méthode permet d'analyser directement certains composés organiques sorbés dans des matrices complexes telles que des échantillons provenant de l'environnement. Dans un premier temps, une impulsion émise par un laser infrarouge désorbe thermiquement les substances à analyser. Un laser ultraviolet pulsé est ensuite utilisé pour les photoioniser par un mécanisme résonnant à deux photons (ionisation multiphotonique amplifiée par résonnance, 1+1 resonanceenhanced multiphoton ionization, 1+1 REMPI). Cette technique particulière d'ionisation évite la fragmentation des molécules et permet la ionisation sélective de certaines molécules en fonction de leur absorption spécifique à la longueur d'onde du laser ultraviolet. La détection des ions est ensuite effectuée au moyen d'un spectromètre de masse à temps de vol pourvu d'un réflectron. Le spectre de masse ainsi obtenu est dominé par les ions parents des constituants de l'échantillon qui absorbent fortement la longueur d'onde du laser UV.

Des applications de la L2MS déjà décrites dans la littérature sont entre autres l'analyse de météorites [7,8], de sols [9], de polymères [10,11], de roches kérogènes [12], de marqueurs tumoraux [13], ou, justement, d'aérosols atmosphériques $[14,15]$. Des mesures quantitatives sont possibles sous certaines conditions $[16,17]$. Par contre, la distinction entre isomères reste très aléatoire en dépit de l'utilisation d'un laser ultraviolet spécial susceptible d'être réglé exactement à la longueur d'onde résonnante d'un isomère donné [18]. 
$A, B$ et $C$ sont 3 analytes avec des spectres d'absorption différents.

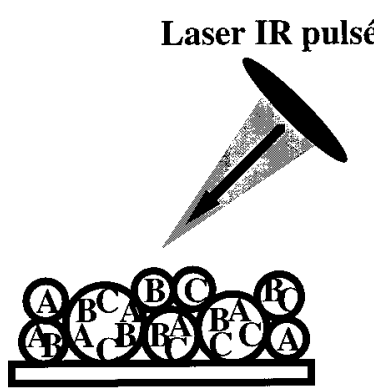

1. Désorption
Spectromètre de masse temps de vol
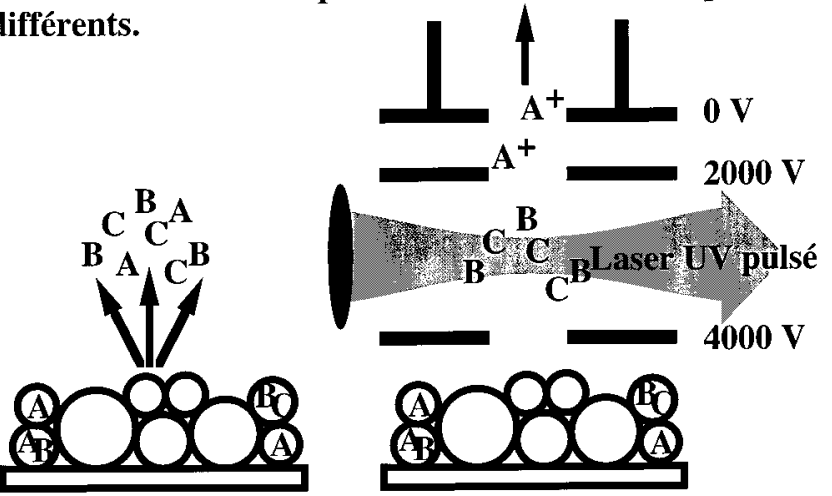

2. Ionisation douce sélective

Figure 1. Principe de la spectrométrie de masse à deux lasers (L2MS). L'exemple montre des particules d'aérosol récoltées sur un filtre en fibres de quartz. (1) L'impulsion émise par le laser infrarouge permet un transfert quantitatif des composés à analyser dans la phase gazeuse. (2) Un laser ultraviolet postionise les molécules. Les ions sont alors analysés au moyen d'un spectromètre de masse à temps de vol.

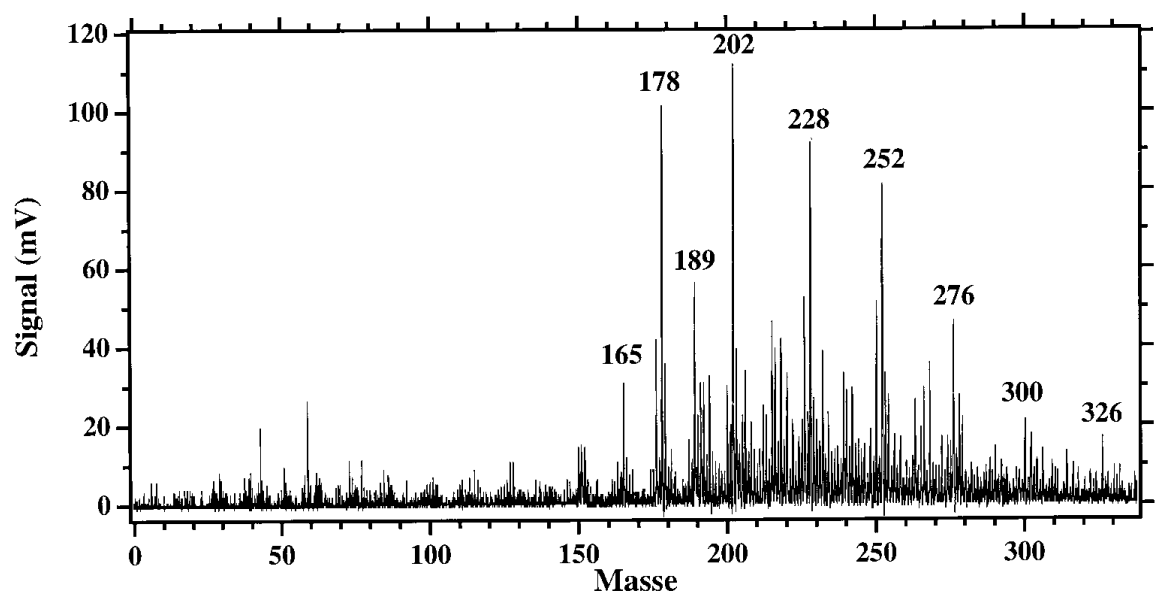

Figure 2. Exemple de spectre L2MS. Tir laser unique sur des particules déposées sur un filtre de fibres de quartz. Les pics à $m / z$ $178,202,228,252,276,300$ et 326 , entre autres, sont attribués à des hydrocarbones aromatiques polycycliques (PAHs, voir Fig. 3). La fragmentation des molécules est presque complètement évitée. Les faibles pics aux masses 43 et 59 correspondent à des fragments dont l'origine est inconnue; ils ne proviennent pas de PAHs, comme l'ont démontré des expériences préliminaires.

Pour l'étude de la composition chimique des aérosols atmosphériques en fonction du temps, la longueur d'onde d'ionisation a été ajustée à $250 \mathrm{~nm}$. Cette longueur d'onde est absorbée particulièrement efficacement par les PACs. La figure 2 montre un exemple typique de spectre L2MS d'aérosol atmosphérique ; on voit que les pics dominants correspondent à des hydrocarbones aromatiques polycycliques (PAHs, voir Fig. 3). La haute sensibilité de la L2MS (de l'ordre de grandeur de l'attomole par tir de laser) ainsi que la quasi inexistance de préparation des échantillons avant d'être mesurés, sont des avantages déterminants qui ont rendu notre étude possible. Des spectres de bonne qualité ont pu être obtenus sur la base d'aérosols récoltés pendant seulement 15 minutes et dont la mesure a duré 5 minutes. Des porte-filtres sur lesquels des filtres en fibres de quartz d'environ $1 \mathrm{~cm}^{2}$ peuvent être montés ont été développés spécifiquement pour cette application. Utilisés pour récolter les aérosols atmosphériques ( $20 \mathrm{~L}$ air/min), ils sont ensuite introduits tels quels dans notre appareil L2MS pour être analysés immédiatement.

La figure 4a montre le développement du signal observé à la masse 202, mesuré en ville de Zurich le 3 février 1998. $\mathrm{Ce}$ signal peut être attribué essentiellement au pyrène $\left(\mathrm{C}_{16} \mathrm{H}_{10}\right)$, puisque des expériences préliminaires ont montré que son isomère, le fluoranthène, est ionisé beaucoup moins efficacement [18]. À titre de comparaison, la figure $4 \mathrm{~b}$ 


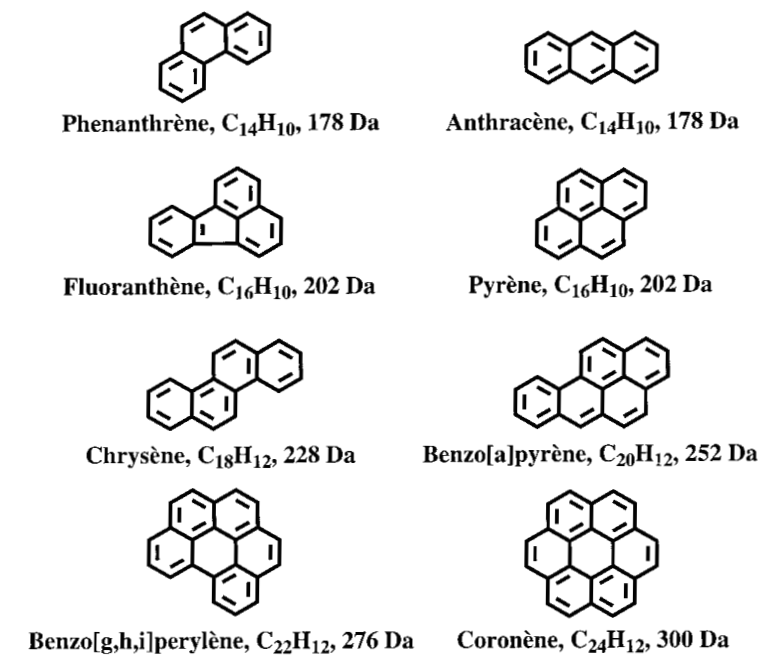

Figure 3 : Liste non-exhaustive de quelques PAHs (pour plus de détails voir [4]).

montre le développement du signal à la masse 290 (benzocyclopentachrysènes, $\mathrm{C}_{23} \mathrm{H}_{14}$, et isomères), mesuré le même jour au même endroit. Â la différence du signal à la masse 202, ce signal est resté globalement constant, et aucune augmentation n'a été observée tôt le matin.

Nos résultats indiquent donc que différents PACs ont différentes origines et différents destins. La mesure de variables telles que le carbone élémentaire, le carbone organique, ou même $\Sigma$ (PACs), ne réflète donc pas complètement la composition moléculaire de la surface des particules d'aérosol. Par contre, les résultats livrés par la L2MS apportent des informations moléculaires détaillées, mesurées en fonction du temps, qui permettent de mieux estimer les risques toxicologiques des aérosols ainsi que la réactivité de leurs surfaces.

Diverses études par chromatographie [19,20] ont montré que les composés organiques dominants à la surface des particules dépendent de la source d'émission des aérosols. Des investigations L2MS supplémentaires sont actuellement en cours dans notre laboratoire dans cette optique. Un des buts poursuivis est l'identification puis l'application de traceurs spécifiques qui permettraient d'identifier en temps réel les sources d'émission des aérosols atmosphériques.

\section{Remerciements}

Nous remercions A. Meier et S. Schlatter (Département de l'Environnement et de la Santé de la Ville de Zurich) et C.

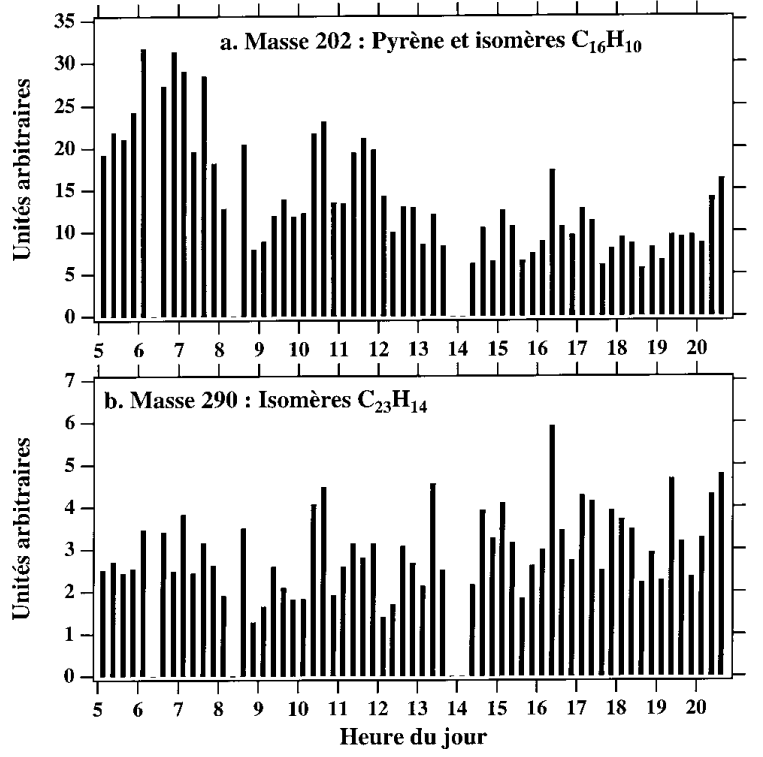

Figure 4. Évolution des signaux aux masses 202 et 290 . Ville de Zurich, 3 février 1998. Chaque point correspond à la moyenne de 50 tirs (écart-type environ $20 \%, S / N>500$ ).

Hüglin (EMPA) pour leur collaboration précieuse, ainsi que S. Haefliger pour ses commentaires sur le manuscrit. Ce projet est soutenu par le Fonds National Suisse de la Recherche Scientifique (Crédit N 510.183).

\section{Références}

1. Pandis, S. N.; Wexler, A. S.; Seinfeld, J. H. J. Phys. Chem. 1995, 99, 9646-9659.

2. Schlesinger, R. B. Inhalation Toxicology 1995, 7, 99-109.

3. Actes de 5th International Aerosol Conference, Edinburgh (publiés dans J. Aerosol Sci., 29, Suppl. 1).

4. Lee, M. L.; Novotny, M. V.; Bartle, K. D. Analytical Chemistry of Polycyclic Aromatic Compounds, Academic Press; New York, 1981.

5. Peltonen, K.; Kuljukka, T. J. Chromatogr. A 1995, 710, $93-$ 108.

6. Voumard, P.; Zhan, Q.; Zenobi, R. Rev. Sci. Instrum. 1993, 25, 3393-3402.

7. Clemett, S. J.; Maechling, C. R.; Zare, R. N.; Swan, P. D.; Walker, R. M. Science 1993, 262, 721-724.

8. McKay, D. S. et al. Science, 1996, 273, 924-930.

9. Dale, M. J.; Jones, A. C.; Pollard, S. J. T.; Langridge-Smith, P. R. R.; Rowley, A. G. Envir. Sci. Technol. 1993, 27, 16931695.

10. Kornienko, O., Ada, E. T.; Hanley, L. Anal. Chem. 1997, 69, 1536-1542.

11. Zhan, Q.; Zenobi, R.; Wright, S. J.; Langridge-Smith, P. R. R. Macromol. 1996, 29, 7865-7871.

12. Zhan, Q.; Zenobi, R.; Buseck, P. R.; Teerman, S. Energy \& Fuels 1997, 11, 144-149.

13. Zhan, Q.; Zenobi, R.; Voumard, P. Anal. Chem., 1994, 65, 3259-3266. 
14. Zhan, Q.; Voumard, P.; Zenobi, R. Rapid. Commun. Mass Spectrom. 1995, 9, 119-127.

15. Dale, M. J.; Jones, A. C.; Pollard, S. J. T.; Langridge-Smith, P. R. R. Analyst 1994, 119, 517-578.

16. Hahn, J. H.; Zenobi, R.; Zare, R. N. J. Am. Chem. Soc. 1987, $109,2842-2843$
17. Haefliger, O. P.; Zenobi, R. Rev. Sci. Instrum. 1998, 69, 18281832.

18. Haefliger, O. P.; Zenobi, R. Anal. Chem. 1998, 70, 2660-2665.

19. Rogge, W. F.; Hildemann, L. M.; Mazurek, M. A.; Cass, G. R.; Simoneit, B. R. T. Environ. Sci. Technol. 1993, 27, 636651.

20. Nielsen, T. Atmosph. Environ. 1996, 30, 3481-3490. 